\title{
ESTUDO PAISAGÍSTICO DA ÁREA ONDE SE ENCONTRA IMPLANTADA A UFRPE, EM GARANHUNS-PE.
}

\author{
Camila Mirelle de Almeida Liberato ${ }^{1}$
}

Maria do Carmo de Albuquerque Braga²

\author{
Amanda Mayara da Costa Lima Santos ${ }^{3}$
}

\begin{abstract}
RESUMO
Entende-se como espaço verde uma espécie de espaço livre que exerce forte influência na funcionalidade urbana e na qualidade de vida da sociedade. Este trabalho objetiva analisar as condições de arborização da área onde se encontra implantada a UFRPE, em Garanhuns/PE UFRPE/UAG, de forma a promover um ambiente saudável e confortável para a comunidade acadêmica e seus visitantes. O presente estudo teve como base a lista de espécies utilizadas na arborização da UFRPE/UAG, e através de visitas in loco percorrendo a unidade realizou-se o censo de arborização, onde se utilizou ficha específica, ou seja, o apêndice. Os critérios adotados foram: estimativa da quantidade de árvores e a classificação das espécies. Foi catalogado e demarcado no mapa as espécies arbóreas que já existiam na área e as implantadas recentemente, com registro fotográfico e classificação das plantas encontradas, relacionado à família, espécie, origem e características do porte. No inventário pode-se observar a ocorrência de cerca de 60 espécies existentes de árvores na área, sendo 33 já existentes e 27 inseridas recentemente, no campus. A partir destas premissas citadas conclui-se que a UFRPE/UAG, para sua implantação, houve consequências devido ao desmatamento. Com isso promoveu-se uma ilha de calor que caso seja realizada a recuperação das espécies vegetais, poderá ser transformado em uma ilha de amenidade climática, de forma a resgatar as espécies nativas e assim também a identidade e a cultura da região.
\end{abstract}

PALAVRAS-CHAVE: Espaço verde. Universidade. Inventário.

\footnotetext{
${ }^{1}$ Graduanda no curso de Agronomia, Universidade Federal Rural de Pernambuco - UFRPE/ Unidade Acadêmica de Garanhuns - UAG. E-mail: mila_liberato@hotmail.com.

${ }^{2}$ Professora adjunta, Universidade Federal Rural de Pernambuco - UFRPE/ Unidade Acadêmica de Garanhuns - UAG. E-mail: mariabraga77@gmail.com.

${ }^{3}$ Graduanda no curso de Agronomia, Universidade Federal Rural de Pernambuco - UFRPE/ Unidade Acadêmica de Garanhuns - UAG. amanda_mayara2@hotmail.com.
} 


\title{
LANDSCAPE STUDY AREA WHERE IS IMPLANTED THE UFRPE, IN GARANHUNS-PE.
}

\begin{abstract}
It understood as a kind of green space free space that exerts a strong influence on urban functionality and quality of life of society. This work aims to analyze the conditions for afforestation of the area where the facility is located UFRPE in Garanhuns / PE UFRPE / UAG in order to promote a healthy and comfortable environment for the academic community and its visitors. This study was based on the list of species used in afforestation of UFRPE / UAG and through site visits covering the unit carried out the census of trees where we used specific form the appendix. The criteria adopted were estimate of the amount of trees and species classification. Was cataloged and marked on the map tree species that existed in the area and deployed recently with photographic recording and classification of plants found, related to the family, species, origin and characteristics of size. In inventory can observe the occurrence of about 60 existing species of trees in the area, with 33 existing and 27 newly entered in the campus. From these premises it follows that cited UFRPE / UAG, for its implementation, there were consequences due to deforestation. With that promoted a heat island that where recovery of plant species is held, can be transformed into an island climate amenity, in order to rescue the native species and thus the identity and culture of the region.
\end{abstract}

KEY WORDS: Green area. University. Inventory.

\section{ESTUDIO DE PAISAJE ZONA DONDE SE IMPLANTÓ EL UFRPE, EN GARANHUNS-PE.}

\section{RESUMEN}

Se entiende como un espacio verde lo espacio libre que ejerce una fuerte influencia en la funcionalidad urbana y la calidad de vida de la sociedad. Este estudio tuvo como objetivo analizar las condiciones para la forestación de la zona donde se encuentre la instalación UFRPE en Garanhuns/PE, con el fin de promover un ambiente sano y confortable para la comunidad académica y de sus visitantes. Este estudio se basó en la lista de las especies utilizadas en la repoblación forestal de la universidad con visitas del campo en la unidad llevando a cabo el censo de los árboles donde solíamos forma especifica, es decir el apéndice. Los criterios adoptados fueron: estimación de la cantidad de árboles, especies e clasificaciones. Fue catalogado las especies arbóreas que existian en la zona y desplegadas recientemente con registro fotográfico y clasificación de las plantas que se encuentran, en relación con la familia, especie, origen y características de tamaño. En el inventario se puede observar la presencia de cerca de 60 especies de árboles existentes en la zona, con 33 existentes y 27 recién entrado en el campus. Entonces se deduce que citan UFRPE / UAG, para su aplicación, las consecuencias son debidas a la deforestación. Con eso promovió una isla de calor que, cuando se lleva a cabo la recuperación de las especies vegetales, se puede transformar en una isla equipamiento climático, con el fin de rescatar a las especies nativas y también la identidad y la cultura de la región.

PALABRAS-CLAVE: Espacio verde. Universidad. Inventario. 
necessidade de manutenção do micro-clima local. As etapas metodológicas seguidas foram:

1 - Estudo teórico e empírico sobre o tema para melhor se definir as funções dos espaços oferecidos pela implantação dos edifícios que compõem a Unidade Acadêmica de Garanhuns, e assim poder realizar a associação entre esses espaços e suas possíveis funções;

2 - Identificação dos novos espaços livres da área onde se encontra implantada a Universidade Federal Rural de Pernambuco, em Garanhuns/PE,por meio do conjunto de plantas que compõem o projeto de arquitetura da Unidade. E logo em seguida iniciaram-se as visitas a campo, no qual se realizou inventário quantitativo visando conhecer a distribuição espacial das espécies arbóreas já existentes no local e das mudas implantadas recentemente na área;

3 - Visita in loco nas áreas externas, caracterização, identificação e análise dos novos espaços destinados ao paisagismo. No qual de posse do mapa, dividiu-se a área em espaços, e procedeu-se a catalogação e demarcação de todas as árvorese mudas existentes nas calçadas e áreas verdes.

O presente estudo teve como base a lista das espécies utilizadas recentemente na arborização da Universidade Federal Rural de Pernambuco. Em continuidade a este trabalho, através de visita in loco percorrendo as repartições da unidade realizou-se um censo da arborização, onde para sua quantificação fez-se uso de formulário adequado para situação constando espaços para descrição de características de folha, tronco, flores e frutos. Os critérios adotados foram à estimativa quantitativa das árvores e a classificação das espécies. Foram inventariadas todas as plantas existentes, com registro fotográfico de algumas e revisão bibliográfica das plantas encontradas, relacionadas á família, nome científico, origem e características do porte e diversidade de espécies.

No inventário realizado observou-se a composição de cerca de 60 espécies existentes na arborização da área, sendo 33 de arbóreas já presentes e 27 de mudas inseridas recentemente, onde se encontra implantada a Universidade Federal Rural de Pernambuco. Quanto às mudas, optou-se por separar as espécies quanto a origem, resultando todas as suas 27 espécies implementadas de origem Nativa e nenhuma de origem exótica, como mostra a tabela 1, abaixo: 
Tabela 1: Inventário de arborização das mudas encontradas na área onde se encontra implantada a Universidade Federal Rural de Pernambuco, em Garanhuns Pernambuco.

\begin{tabular}{|c|c|c|c|c|}
\hline Nome comum & Nome científico & Origem & Família & Característica \\
\hline Abiu & $\begin{array}{l}\text { Pouteria caimito (Ruiz \&Pav.). } \\
\text { Radlk. }\end{array}$ & Nativa & Sapotaceae & Porte médio \\
\hline Aroreira-da-praia & SchinusterebinthifoliusRaddi & Nativa & Anacardiaceae & Porte médio \\
\hline Aticum & AnnonamontanaMacfad & Nativa & Annonaceae & Porte médio \\
\hline Babatenon & $\begin{array}{c}\text { Abaremacochliacarpos (Gomes) } \\
\text { Barneby\& J.W. Grimes }\end{array}$ & Nativa & Fabaceae & Porte médio \\
\hline Bordão-de-velho & $\begin{array}{c}\text { Samanea tubulosa (Benth.) } \\
\text { Barneby\&J.W.Grimes }\end{array}$ & Nativa & Mimosaceae & Grande Porte \\
\hline Peito de Pombo & TapiriraguianensisRaddi & Nativa & Anacardiaceae & $\begin{array}{l}\text { Porte médio a } \\
\text { grande }\end{array}$ \\
\hline Goajurú & Chrysobalanusicaco L. & Nativa & Chrysobalanaceae & Porte médio \\
\hline Ingazinho & IngacapitataDesv. & Nativa & Mimo & $\begin{array}{l}\text { Pequeno } \\
\text { Porte }\end{array}$ \\
\hline $\begin{array}{l}\text { Ingá-do- } \\
\text { brejo }\end{array}$ & Inga sp. & Nativa & Mimosaceae & Porte médio \\
\hline Ipê-roxo & Tabebuia cf. heptaphylla(Vell.) & Nativa & Bignoneaceae & Porte alto \\
\hline Jaquarana & Albiziapedicellaris (DC.) L.Rico. & Nativa & Mimosaceae & Porte médio \\
\hline Jucá. & $\begin{array}{c}\text { Libidibiaferrea (Mart. ex. Tul.) } \\
\text { L.P.Queiroz }\end{array}$ & Nativa & Fabaceae & Grande porte \\
\hline Japaranduba & Gustavia augusta $L$. & Nativa & Lecythidaceae & Porte médio \\
\hline Marí & Geoffroeastriata (Willd.) Morong & Nativa & Leguminoseae & Porte médio \\
\hline Munguba & $\begin{array}{c}\text { Eriothecagracilipes (K. Schum.) A. } \\
\text { Robyns }\end{array}$ & Nativa & Bombacaceae & Porte médio \\
\hline Mutambo & GuazumaulmifoliaLam. & Nativa & Malvaceae & Porte médio \\
\hline Oiti-da-prata & Licania tomentosa (Benth.) Fritsch & Nativa & Chrysobalanacea & Grande porte \\
\hline $\begin{array}{l}\text { Pau-de- } \\
\text { jangada }\end{array}$ & Apeibatibourbouaubl. & Nativa & Tiliaceae & Grande porte \\
\hline Pitombeira & Talisiaesculenta (Cambess.) Radlk. & Nativa & Sapindaceae & Grande porte \\
\hline $\begin{array}{l}\text { Pitombinha- } \\
\text { de-macaco }\end{array}$ & Pouteriabangii (Rusby) T.D.Penn. & Nativa & Sapotaceae & Grande porte \\
\hline Pitanga & Eugenia uniflora $L$. & Nativa & Mirtaceae & $\begin{array}{l}\text { Pequeno a } \\
\text { médio porte }\end{array}$ \\
\hline Pororoca & ClusiaNemorosa G. Mey. & Nativa & Clusiaceae & Porte médio \\
\hline Pau-rei & Pterygota brasiliensis Allemão & Nativa & Anacardiaceae & Grande porte \\
\hline Salgueiro & AegiphilapernambucensisMoldenke & Nativa & Lamiaceae & Porte médio \\
\hline Tamanqueiro & Simarouba amara Aubl. & Nativa & Simaroubaceae & Grande porte \\
\hline Tamboril & $\begin{array}{c}\text { Enterolobiumcontortisiliquum (Vell.) } \\
\text { Morong }\end{array}$ & Nativa & Mimosaceae & Grande porte \\
\hline Ubaia & Eugenia sp. & Nativa & Mirtaceae & Porte médio \\
\hline
\end{tabular}

Fonte: BRAGA, CARVALHO, CEPAN, GIOCOMETTI, LORENZI, MILANO, 2014

Através da visita in loco foi possível catalogar e demarcar as 27 espécies de mudas, que foram distribuídas em quantidade e tamanho de forma aleatória no campus, tendo um total de 690 mudas reconhecidas e plantadas na unidade 


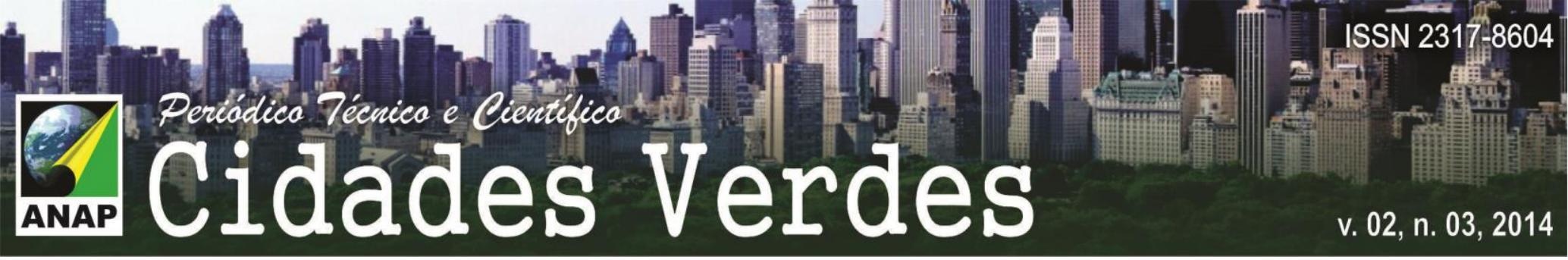

acadêmica. Com base no projeto de implantação da Universidade na área, fez-se a identificação da localização destas mudas na Unidade Acadêmica:

Figura 1: Mapeamento das mudas de espécies arbóreas já introduzidas na unidade. (A) Bloco sala de aula 01, (B) Bloco sala de aula 02, (C) Bloco sala de aula 03, (D) Bloco laboratórios, (E) Bloco administrativo. Fonte: Departamento de engenharia e planejamento da Universidade Federal Rural de Pernambuco - Campus Garanhuns (UAG).

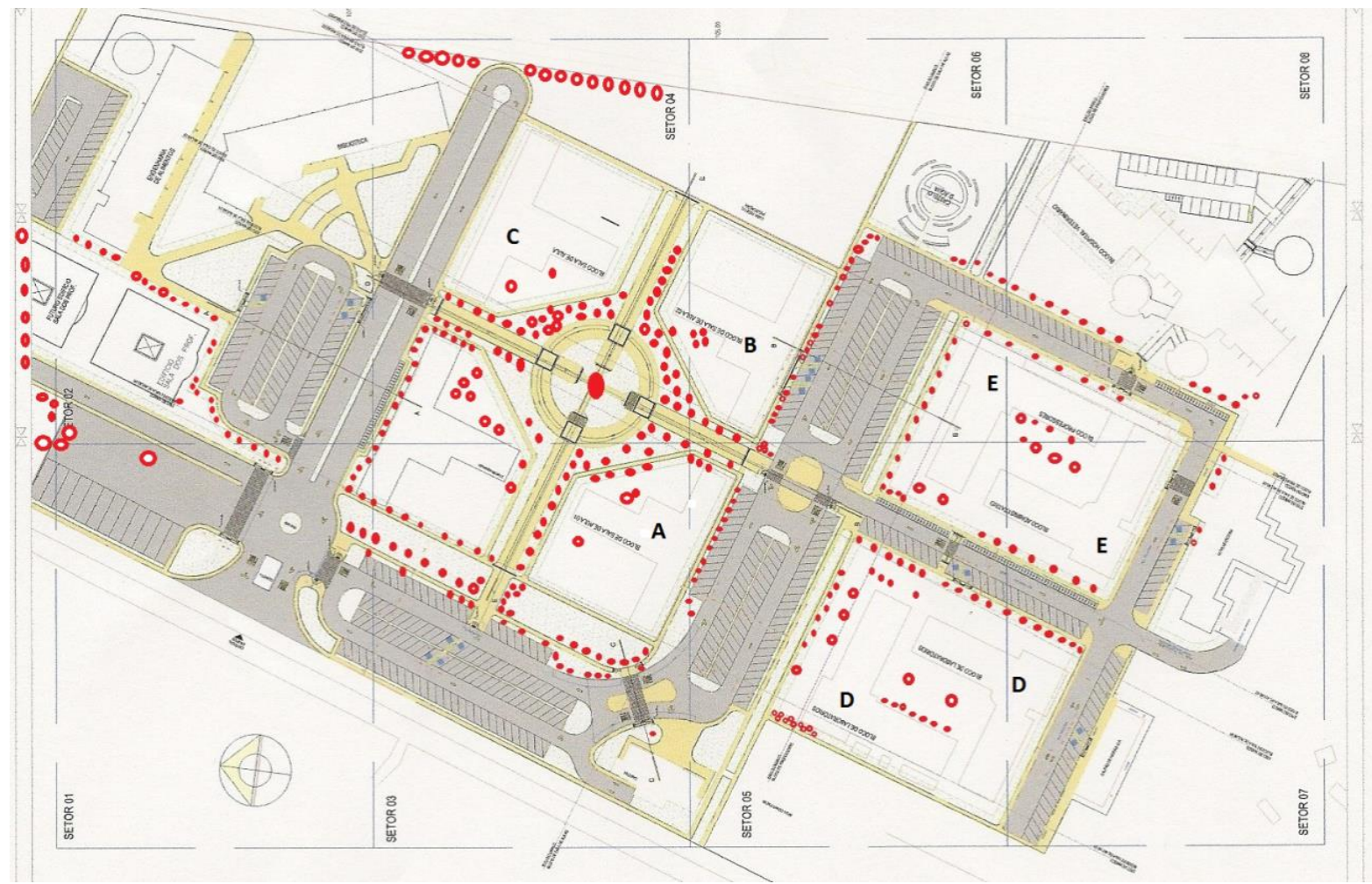

Fonte: Equipe da pesquisa, com base no projeto de implantação da Universidade na área de estudo, 2014.

Já com relação as espécies Arbóreas, foram levantadas cerca de 33 espécies, das quais, 23 foram identificadas e catalogadas. Optou-se também nesse caso, catalogar as espécies quanto a origem, resultando 4 espécies de origem Nativa e 16 de origem exótica, das que foram catalogadas, como mostra a tabela 2 abaixo: 
Tabela 2: Inventário de arborização das arbóreas encontradas na área onde se encontra implantada a Universidade Federal Rural de Pernambuco, em Garanhuns Pernambuco.

\begin{tabular}{|c|c|c|c|c|}
\hline Nome comum & Nome científico & Origem & Família & Característica \\
\hline Pitangueira & Eugenia unifloraL. & Nativa & Myrtaceae & $\begin{array}{l}\text { Pequeno a } \\
\text { médio porte }\end{array}$ \\
\hline Pau-brasil & Caesalpineaechinata & Nativa & Caesalpiniodeae & $\begin{array}{c}\text { Médio a } \\
\text { grande porte }\end{array}$ \\
\hline Goiabeira & Psidiumguajava L. & Nativa & Myrtaceae & Porte médio \\
\hline $\begin{array}{l}\text { Timburi-do- } \\
\text { cerrado }\end{array}$ & $\begin{array}{c}\text { Enterolobiumgummiferum(Mart.) } \\
\text { J.F. Macbr. }\end{array}$ & Nativa & Mimosoideae & Porte médio \\
\hline Castanhola & TerminaliacatappaL. & Exótica & Combretaceae & Grande Porte \\
\hline Amoreira & Morus nigraL. & Exótica & Moraceae & Porte médio \\
\hline $\begin{array}{l}\text { Jasmin } \\
\text { Laranja }\end{array}$ & Murrayapaniculata(L.) Jacq.. & Exótica & Rutaceae; & $\begin{array}{l}\text { Pequeno a } \\
\text { médio porte }\end{array}$ \\
\hline Flamboiã & $\begin{array}{c}\text { Delonix regia (BojerexHook.) } \\
\text { Raf. }\end{array}$ & Exótica & Leguminoseae & $\begin{array}{c}\text { Médio a } \\
\text { grande porte }\end{array}$ \\
\hline Cica & CycascircinalisL. & Exótica & Cycadaceae & $\begin{array}{l}\text { Pequeno a } \\
\text { médio porte }\end{array}$ \\
\hline Eritrina & ErythrinavariegataL. & Exótica & Papilionoideae & Grande porte \\
\hline Jaguarana & Albiziapedicellaris (DC.) L. Rico. & Nativa & Mimosaceae & Porte médio \\
\hline Brasileirinho & $\begin{array}{c}\text { Erythrina indica Lam. var. picta } \\
\text { Hort. }\end{array}$ & Exótica & Papilionoideae & $\begin{array}{l}\text { Pequeno a } \\
\text { médio porte }\end{array}$ \\
\hline $\begin{array}{l}\text { Chuva-de- } \\
\text { ouro }\end{array}$ & Cassia fistula L. & Exótica & Caesalpinioideae & $\begin{array}{c}\text { Médio a } \\
\text { Grande porte }\end{array}$ \\
\hline Eucalipto & Eucalyptus sp. & Exótica & Myrtaceae & Grande porte \\
\hline Jaqueira & ArtocarpusheterophyllusLam. & Exótica & Moraceae & Grande porte \\
\hline Mangueira & Mangifera indica L. & Exótica & Anacardiaceae & $\begin{array}{c}\text { Médio a } \\
\text { grande porte }\end{array}$ \\
\hline Pinheiro & Pinus sp. & Exótica & Pinaceae & Grande porte \\
\hline Algaroba & Prosopisjuliflora (Sw.) DC. & Exótica & Mimosoideae & Grande porte \\
\hline Ciriguela & Spondiaspurpurea L. & Exótica & Anacardiaceae & Médio porte \\
\hline Abacateira & Persea americana Mill. & Exótica & Lauraceae & $\begin{array}{c}\text { Médio a } \\
\text { grande porte }\end{array}$ \\
\hline Graviola & AnnonamuricataL. & Exótica & Annonaceae & $\begin{array}{l}\text { Porte pequeno } \\
\text { a médio }\end{array}$ \\
\hline
\end{tabular}

Fonte: EMBRAPA, LORENZI, 2014

Através da visita in loco, foram demarcadas 210 arbóreas divididas em 33 espécies distribuídas em todo o campus da Universidade. Com base também no projeto de implantação da Universidade na área, fez-se a identificação da localização destas arbóreas na Unidade Acadêmica: 


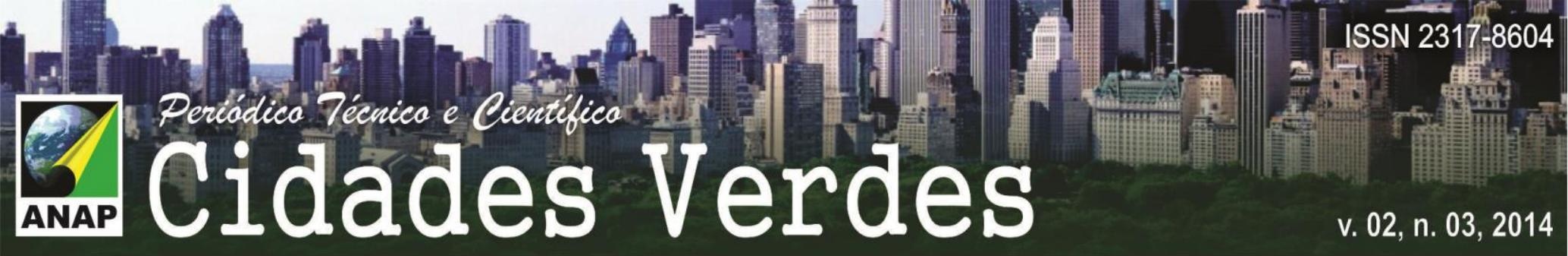

Figura 2: Mapeamento de espécies arbóreas presentes na unidade. (A) Bloco sala de aula 01,

(B) Bloco sala de aula 02, (C) Bloco sala de aula 03, (D) Bloco laboratórios, (E) Bloco administrativo. Fonte: Departamento de engenharia e planejamento da Universidade Federal Rural de Pernambuco

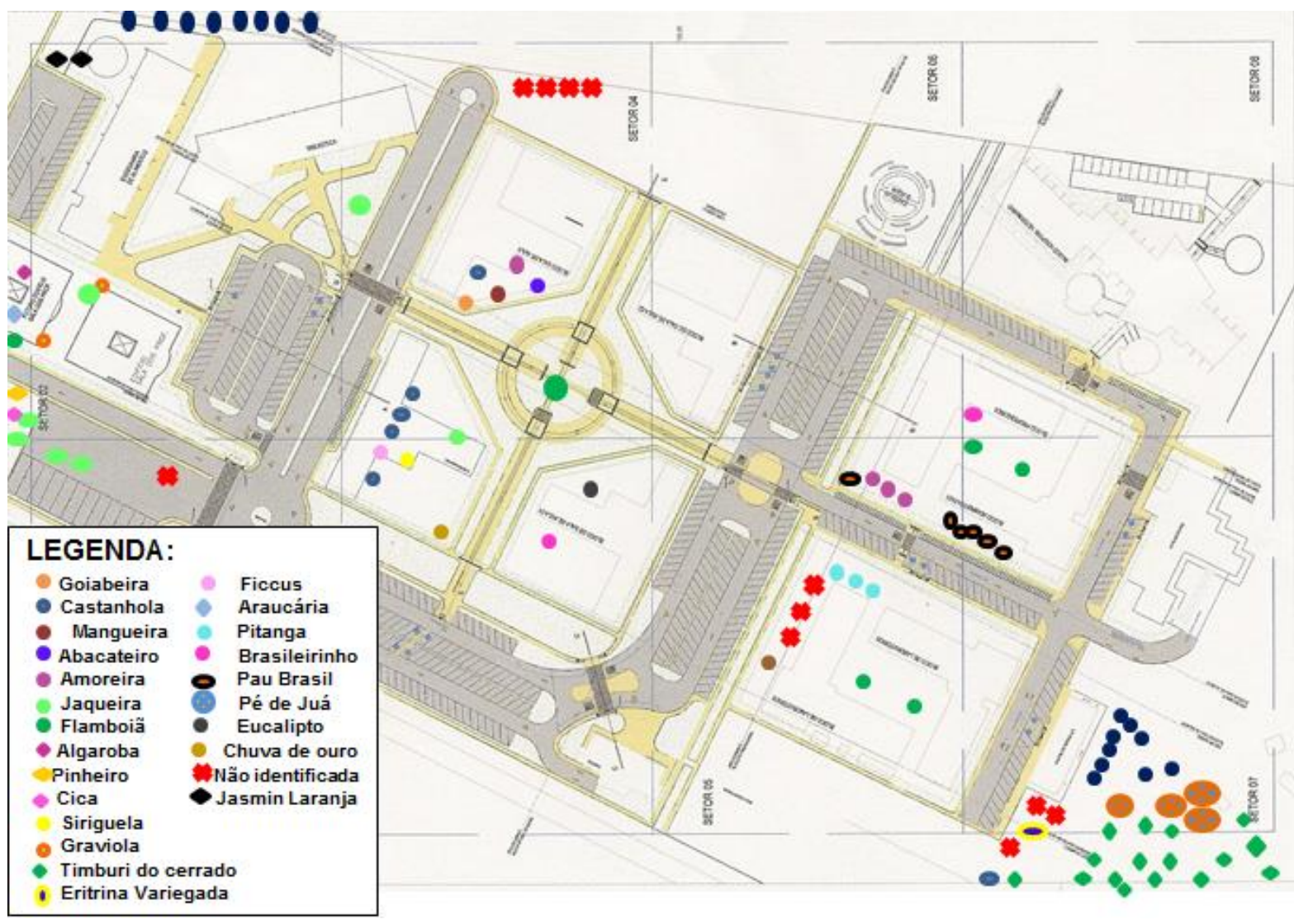

Fonte: Equipe da pesquisa, com base no projeto de implantação da Universidade na área de estudo, 2014.

\section{CONCLUSÃO}

Através do levantamento da composição arbórea presente na área onde se encontra implantada a Universidade Federal Rural de Pernambuco, observou-se a escassez de arborização existente no campus. Além disso, a arborização existente encontra-se mal distribuída e de forma localizada, em determinadas áreas. Observou-se ainda que entre as espécies catalogas a grande maioria é de origem exótica, sendo essas espécies adaptadas às condições ambientais da região, mas não proporcionam os benefícios que uma mata nativa oferece.

Quanto as mudas catalogadas, todas são de origem nativa, sendo um aspecto positivo quanto a correlação com a visão da proposta do projeto. No entanto, é uma solução a longo prazo quanto a implantação de áreas verdes. Para 


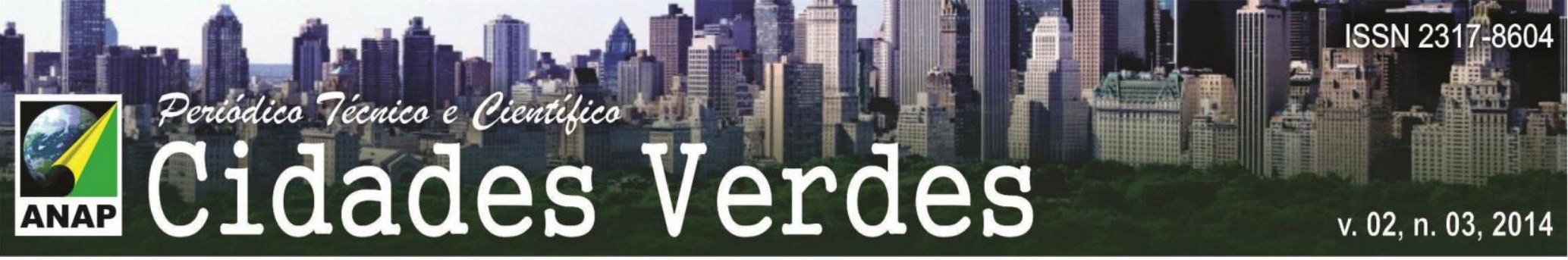

BELLÉ, Soeni; Apostila de Paisagismo. Bento Gonçalves: Instituto Federal de Educação, Ciência e Tecnologia do Rio Grande do Sul - IFRS, 2013.

Instituto do Meio Ambiente e dos Recursos Naturais Renováveis; Espécies Nativas da Flora Brasileira de Valor Econômico atual e Potencial. Brasília, DF: 2011.

Rodolfo Júnior R, F.; Melo, R. R.; Cunha, T. A.; Stangerlin, D. M. (2008). Análise da arborização urbana em bairros da cidade de Pombal no Estado da Paraíba. Revista da Sociedade Brasileira de Arborização Urbana, v. 3, n. 4, p. 3-19.

Lorenzi, H. et al. (2003). Árvores exóticas no Brasil: madeiras, ornamentais e aromáticas. Nova Odessa: Instituto Plantarum,v. 1. 